der Vergleich mit der Parallel- und Sekundärüberlieferung und die unverhältnismäßig vielen, im Laufe der Zeit gemachten Änderungsvorschläge verschiedener Gelehrter zeigen, daß diese Überlieferung nicht gut ist ${ }^{1}$, habe ich nach Möglichkeit den Text von $b$ belassen, solange er verständlich und nicht falsch ist ${ }^{2}$. Trotzdem war eine große Zahl von Änderungen nicht zu umgehen: S. 5,$4 ; 8,28 ; 9,24 ; 15,4.13$; 16,$15 ; 17,4$. 10 (bis). 11. $18 ; 18,6$. 7. 14. 18. $24 ; 21,20$. $26 ; 23,15$; 24,$21 ; 25,15 ; 27,4.17 ; 28,4 ; 31,3.12 ; 32,16.17 ; 34,6 ; 42,4$. 17. 29; 43,$19 ; 45,11 ; 46,5 ; 49,4$. 18. $21 ; 50,19 ; 52,23 ; 53,5 ; 55,20 ; 57,19$. $30 ; 62,24 ; 64,6 ; 68,8 ; 74,6$. $14 ; 80,17 ; 81.5$. 14. 20. 29. 31; 82,1. 2 . $3 ; 83,20 ; 85,14 ; 87,12.15 .16 ; 89,8.19 ; 96,27 ; 100,3$. 7. 29. 30. 32; $105,2.11$ (bis); 107, 20;110,23; 111, 3. 8; 113,4. 11. 14; 114, 5. 11. 27 ; 115,3.9. 17. $24 ; 119,2$. 11. $30 ; 122,26 ; 126,3.13 .19 ; 127,4.17$; 129,$15 ; 133,21 ; 136,2 ; 138,12.21 ; 139,20-23 ; 140,3.21 ; 141,3$; $143,7.12 ; 145,11$. 15. 20. $21 ; 146,1 ; 147,23 ; 150,18 ; 151,2$.

\title{
Testimonia veterum
}

Überlieferung und Testimonien sind - wenn man die späteren $\mathrm{Ab}$ schriften abrechnet - für solch ein im Hinblick sowohl auf den Verfasser als auch auf Gegenstand und Inhalt interessantes Werk wie die $\mathrm{VC}$ nicht sehr zahlreich. Bei den Testimonien basieren nur die Kirchenhistoriker des 4. und 5. Jahrhunderts, Gelasius von Caesarea, Socrates, Sozomenus und Theodoret, weiter der gelehrte Photios und vielleicht das Carmen Ebedjesu auf eigener Kenntnis der VC. Nur wenige der hagiographischen Viten haben Parallelen zur VC. Von diesen wenigen Viten wiederum haben nur zwei, nämlich $\mathrm{BHG} \mathrm{Nr}$. 363 und $369 \mathrm{~g}$, die VC direkt benutzt, während die anderen ihre Kenntnis aus den Kirchengeschichten des Gelasius von Caesarea und des Theodoret entnommen haben. Beide Viten sind in Konstantinopel entstanden; die eine auf Grund wissenschaftlicher Intentionen (BHG 363), die andere als persönliches Anliegen eines Schreibers oder eines Klosters (BHG 369g). Nur die erste dieser beiden Viten fand weitere Verbreitung. Die Handschriftengrundlage ist schmal; denn nur fünf Handschriften kommen für die Textkonstituierung in Frage, wovon nur eine - sie ist nicht die durchweg beste - aus dem 10. Jahrhundert, die anderen aus dem 12. Jahrhundert und späterer Zeit stammen. BHG $369 \mathrm{~g}$ ist nur in einer Handschrift überliefert.

Für diese schlechte Überlieferung und Bezeugung gibt es mehrere Gründe. Dem wichtigsten begegnen wir im Ansatz schon bei Sokrates (siehe unten S. XXIX), sehr deutlich dann in der Beurteilung derVCdurch

1 Einige der Fehler von $b$ können durchaus auf den Archetyp zurückgehen.

2 Siehe auch unten S. LXVI. 
Photios (siehe unten S. XXXI). Der theologische Gehalt der VC wird hier einer Kritik unterzogen. Euseb galt vielfach nicht als orthodox, sondern als Arianer 1. Zwar wurden seine historischen, nicht aber seine theologischen Werke geschätzt. Und wie das Urteil des Photios zeigt, rechnete die VC mehr zu den Schriften, die die falschen theologischen Ansichten des Euseb enthalten. So enthält auch die älteste Handschrift der VC, nämlich V, nur die VC und die Oratio, wogegen die späteren Handschriften die VC mit der Hist. Eccl, zuammen überliefern. Hier zeigt sich die Anbahnung einer großzügigeren Auffassung.

Die theologische Abwertung der Schriften des Euseb, speziell der VC, bedingte das geringe Interesse an der VC und die Erschwerung ihrer Verbreitung. Sehr aufschlußreich ist in unserem Zusammenhang die Bemerkung des Patriarchen Germanos I. (715-730) in seiner

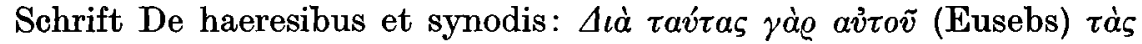

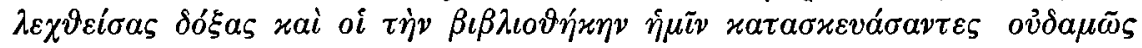

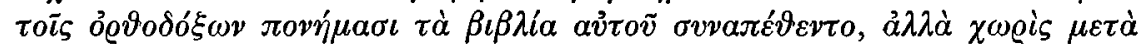

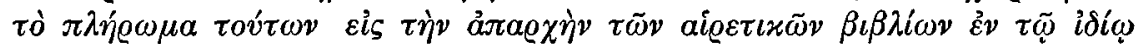

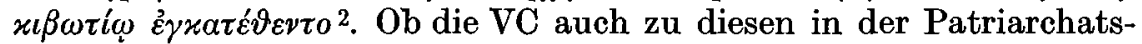
bibliothek separat aufbewahrten Werken gehörte, kann man nur vermuten. Es würde aber die geringe Überlieferungsbreite am besten erklären; denn auch die übrigen Bibliotheken des Patriarchats dürften ähnlich verfahren sein. Die Vermutung wird durch die Tatsache gestützt, daß der sehr belesene und an Quellen für sein Geschichtswerk sehr interessierte Nikephoros Kallistos Xanthopulos die VC nicht direkt benutzt und gekannt hat. Er wertete die Patriarchatsbibliothek aus. Offensichtlich war die VC hier im 14. Jahrhundert nicht zugänglich.

Der zweite wichtige Grund ist, daß das Konstantinbild der VC in vielem nicht den späteren Anforderungen entsprach. Gerade diejenigen Legenden, auf die man später besonderen Wert legte, enthielt sie nicht (zum Beispiel : Gründung Konstantinopels, Helenalegenden mit Kreuzauffindung, Silvester- und Tauflegenden, Kindheitsgeschichten Konstantins). Es fehlte das undifferenzierte Heiligenbild, das man brauchte. Hier sprangen die vielen späteren hagiographischen Konstantinviten ein, die nur das Legendenbild aufnahmen, in den meisten Fällen die Schrift Eusebs nicht benutzten und sie so weitgehend verdrängten.

Fragen wir nach den Zentren, in denen die VC erhalten war, in der Zeit, aus der wir noch keine Handschriften besitzen, so deuten die Erwähnungen bei den alten Kirchenhistorikern, bei Photios und die Verwendung in den Viten BHG 363 und $369 \mathrm{~g}$ auf Konstantinopel. Die

1 Vgl. F. Winkelmann, Die Beurteilung des Eusebius von Cäsarea und seiner Vita Constantini im griechischen Osten, in: Byzantinistische Beiträge, hrsg. von J. Irmscher, Berlin 1964, 91-119.

2 Migne PG 98, 53 A. 
Erwähnung im Carmen Ebedjesu zeigt, daß das Werk auch im syrischpalästinensischen Raum verbreitet war. Man wird hier in erster Linie an die Bibliothek in Kaisareia/Pal. denken, die aber schon beim Arabereinfall 638 zerstört wurde. Wir wissen nicht, wo die Handschriften VJNAB geschrieben wurden. Es würde aber kaum verwundern, wenn ihr Archetyp in Konstantinopel lag.

$\mathrm{Zu}$ den folgenden Testimonien vergleiche man auch den Abschnitt „Die Sekundärüberlieferung“" oben S. XIX ff. ${ }^{1}$

Sozomenus h. e. I 3, $2^{2}$ wird auf Eusebius, allerdings ohne Nennung einer Schrift, verwiesen. Doch folgt die Erzählung der Kreuzesvision Konstantins. Es ist also die VC gemeint.

Theodoret h. e. I 13, $1^{3}$ leitet ein Zitat aus der VC so ein: ... xai

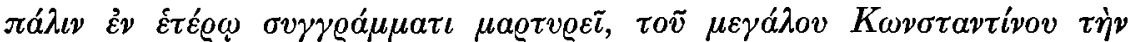

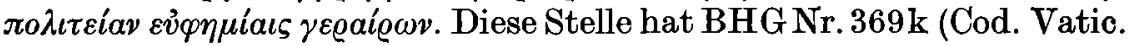
gr. 1991, fol. 9aII) übernommen.

Socrates hat die meisten direkten Verweise auf die VC. Er hat in der 1. Auflage seines Werkes die VC nur über das Werk des Gelasius v. Caes. kennengelernt, diese Quelle aber für die 2. Auflage selbst benutzt und zumindest die genauen Hinweise auf die VC eingesetzt, wenn er sie nicht schon wie I 8, 4. 20 aus dem Werk des Gel. v. Caes. übernommen hat4. I 1, ${ }^{5}$ wird eine Charakteristik der VC gegeben:

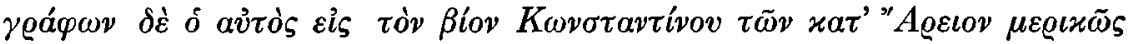

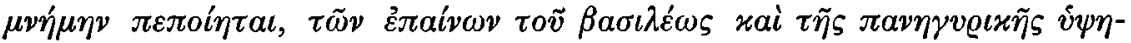

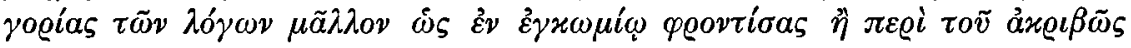

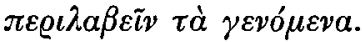

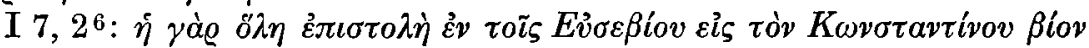

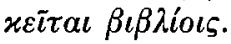

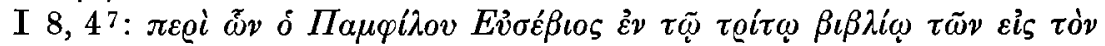

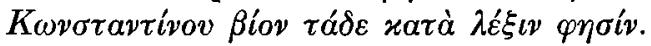

I 8,12 wird das Ende des Zitats mit einem Verweis auf Eusebius angegeben.

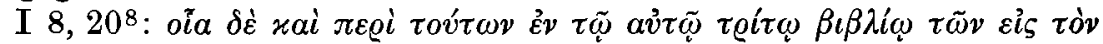

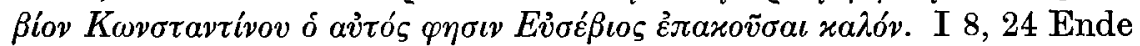
des Zitats mit dem Verweis auf Eusebius.

1 Zum Fehlen eines Testimoniums in De viris ill. des Hieronymus vgl. F. Winkelmann, Zur Echtheitsfrage der Vita Constantini des Eusebius von Cäsarea, Studii Clasice 3, 1961, 405-412.

${ }^{2}$ GCS 50,11, 13 ff. $\quad 3$ GCS 19, 54, 8-10. Siehe unten zu S. 88, 3 .

4 Siehe oben S. XX Anm. 3. 5 Hussey I, 1, 11-2, 4.

6 S. 26, 14f. Socrates hat diese Notiz in der 2. Auflage angebracht, die Urkunde aber aus Gel. Caes. entnommen (siehe unten zu S. 75, 18).

7 S. 36, 5-7. Socr. hat diese Notiz aus Gel. Caes. entnommen (siehe unten zu S. 84,8$)$.

8 S. 41, 18-42, 2. Socr. hat diese Notiz aus Gel. Caes. entnommen (siehe unten zu S. 88,5$)$. 
Indirekte Verweise auf die VC nur mit Nennung des Namens des Eusebius auch I 8, 271, I 16, 42, I 23, $6^{3}$.

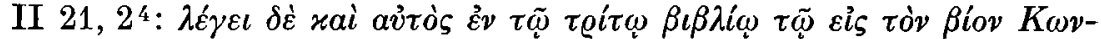

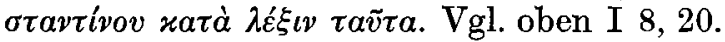

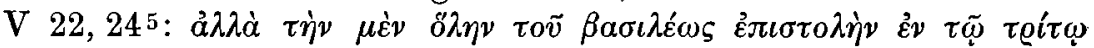

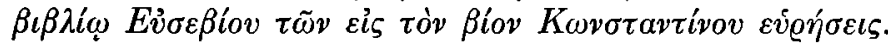

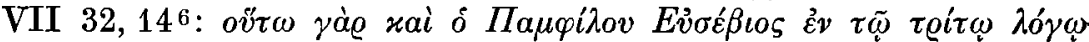

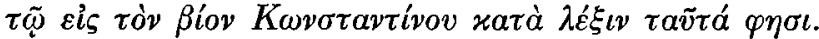

Gelasius von Cyzicus hat zwei in derselben Form bei Socr. vorkommende Erwähnungen und Zitate:

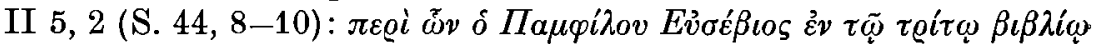

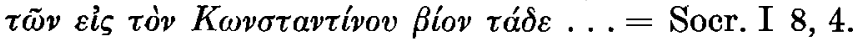

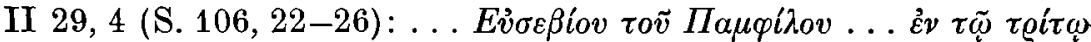

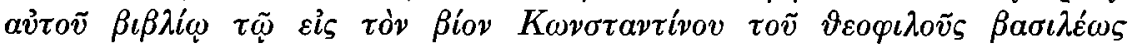
$=$ Socr. I 8, 20 .

Socrates und Gelasius von Cyzicus haben diese Notizen gleichermaßen von Gelasius von Caesarea abgeschrieben ${ }^{7}$.

Leontius von Jerusalem (Anfang 6. Jahrhundert): $\dot{\omega} \varsigma \delta \eta \lambda o i ~ E v ̉ \sigma \varepsilon ́ \beta \iota s$

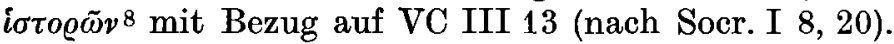

Theophanes, Chronographie (Anfang 9. Jahrhundert): $\pi \varepsilon \varrho i$ $\tilde{\nu} \nu \mu \varepsilon-$

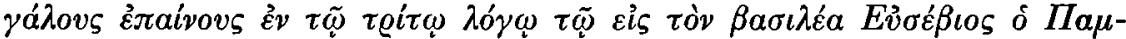

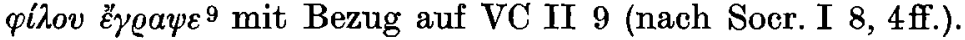

Nicetas Choniata, Thesaurus orthod. fidei V 6 (12./13. Jahrhundert): Eusebius autem Pamphili libro tertio De vita Constantini imperatoris mit Bezug auf VC III 7, 210 (nach Socr. I 8, 4ff.) und V 7: Atque Eusebius quidem libro De Constantini vita tertio se primum verba in synodo fecisse testatur 11 mit Bezug auf VC III 11. Die Quelle läßt sich nicht feststellen.

Nicephorus Callistus Xanthopulus hat in seiner Kirchengeschichte (Anfang 14. Jahrhundert) fast alle VC-Zitate und -Erwähnungen aus Socrates abgeschrieben:

1 S. 44,12 .

2 S. 104, 1-5.

3 S. 131, 12f

4 S. 233,11f. Siehe dazu unten zu S. 88, 5 und 13. Auch diese Notiz stammt wie die I 8, 20 aus Gel. Caes.

5 S. 628, 7-9. Siehe dazu unten zu S. 92, 1 und 89, 17. Die Notiz hat Socr. in der 2. Auflage zugefügt.

6 S. 807, 16-808, 1. Siehe dazu unten zu S. 101, 24. Die Notiz stammt aus eigener Kenntnis des Socrates.

7 Vgl. den oben S. XX Anm. 3 erwähnten Titel.

8 Migne PG 86, 2, $1884 \mathrm{~A}$.

9 Migne PG 108, $97 \mathrm{~B}$.

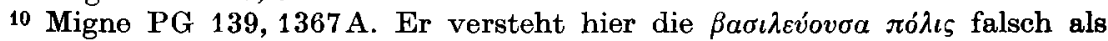
Konstantinopel.

11 Migne PG 139, 1367 B. 


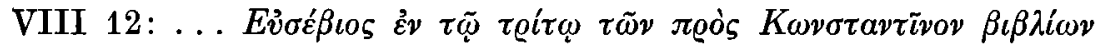

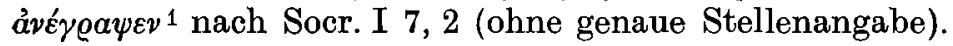

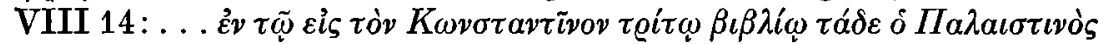

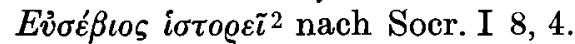

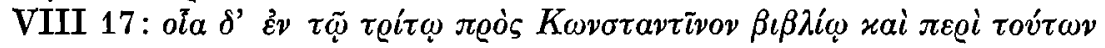

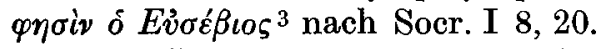

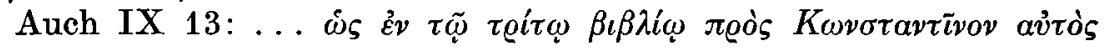

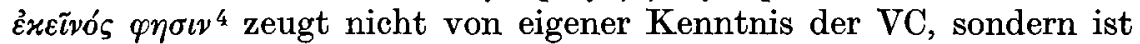
nur aus Nic. VIII 14 herübergenommen, dessen Quelle ja Socr. I 8, $4-11$ ist.

VI 37 gibt ein Schriftenverzeichnis und eine Würdigung des Euse-

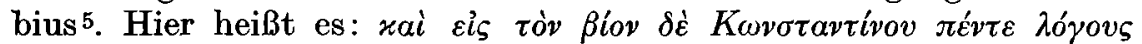
$\dot{\varepsilon}^{\xi} \xi \varepsilon \delta \omega \varkappa \varepsilon$. Diese Bemerkung beruht nicht auf eigener Kenntnis der $\mathrm{VC}$.

Verweise auf Eusebius mit folgenden Parallelen zum VC-Text, jedoch ohne direkte Erwähnung der VC, findet man in zwei hagiographischen Viten:

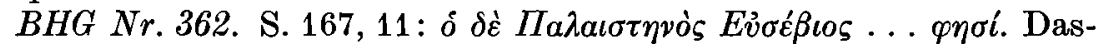
selbe auch in BHG Nr. 369 (Cod. Vindob. Hist. gr. 104, 24b).

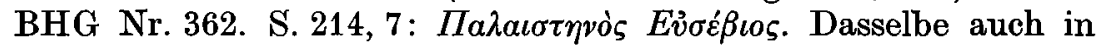
BHG Nr. 369 (Cod. Vindob. Hist. gr. 104, 49b).

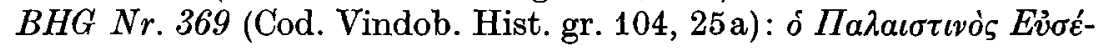

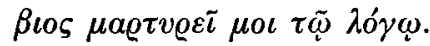

Die Verweise beruhen nicht auf eigener Kenntnis der VC (siehe oben S. XXIII).

Sehr wichtig ist Photius, Bibliotheca cod. 127, mit herber Kritik des Stiles und des Inhaltes der VC8:

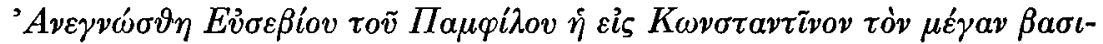

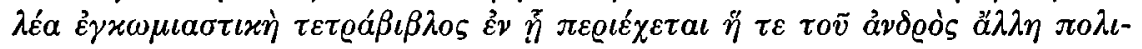

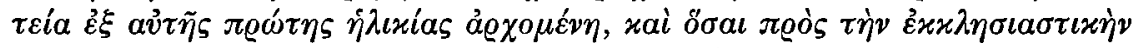

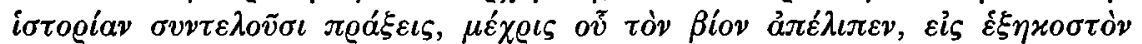

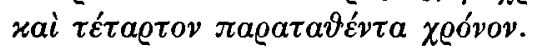

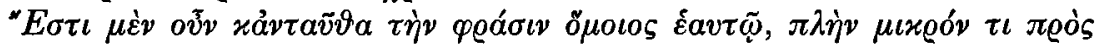

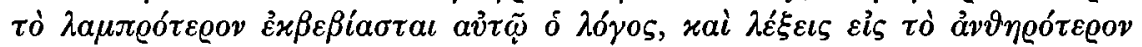

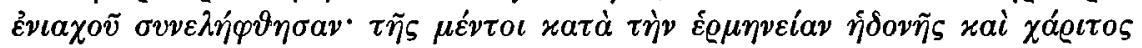

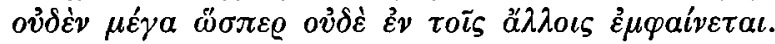

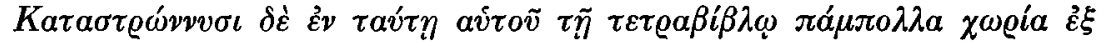

1 Ducaeus I 556ff. (Migne PG 146, 53 A).

2 Ducaeus I $561 \mathrm{f}$. (Migne PG 146, 60 C).

3 Ducaeus I 567f. (Migne PG 146, 69 C).

4 Ducaeus I 651 A (Migne PG 146, 264 A).

5 Ducaeus I 435f. (Migne PG 145, 1204C-1205C).

6 Migne PG 145, 1205 A.

7 Vgl. Byzantinist. Beiträge, hrsg. von J. Irmscher, Berlin 1964, 108.

8 Ed. R. Henry, II, Paris 1960, 99-101. 


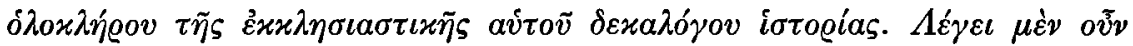

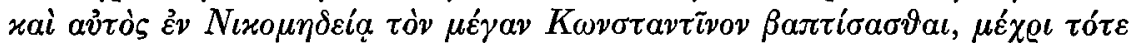

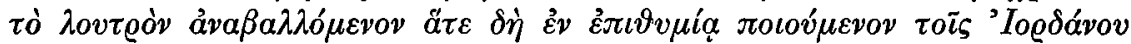

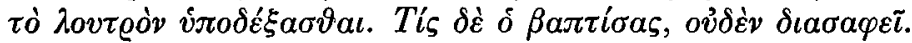

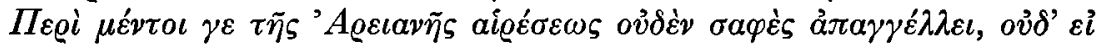

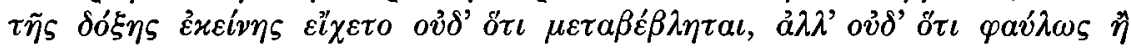

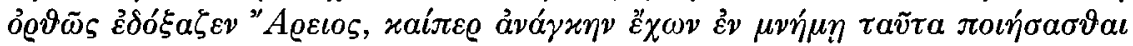

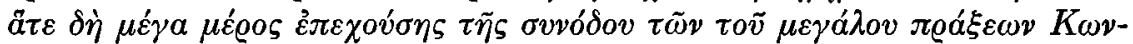

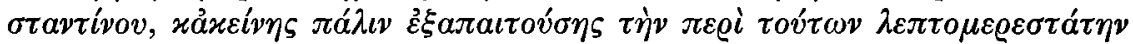

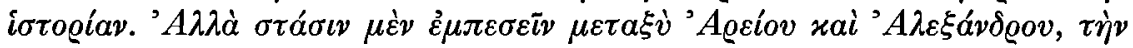

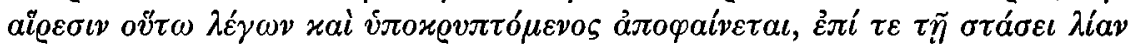

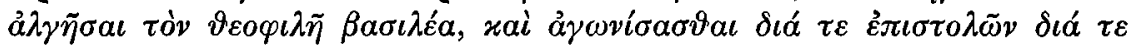

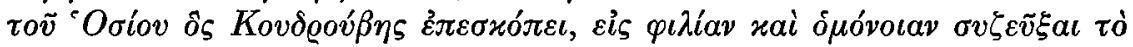

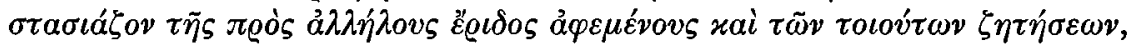

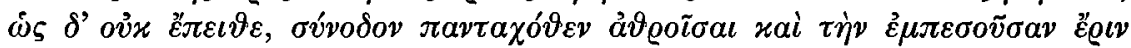

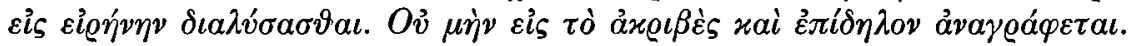

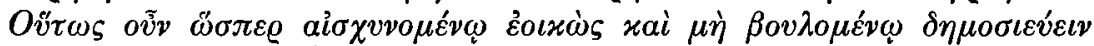

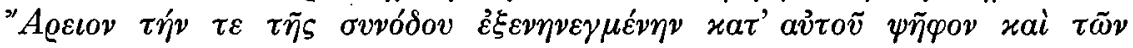

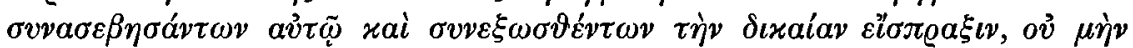

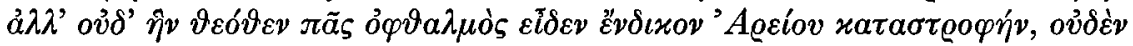

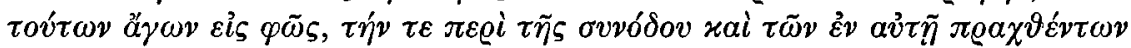

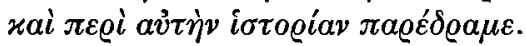

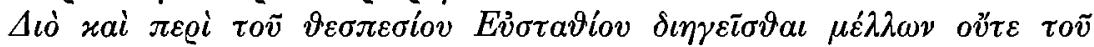

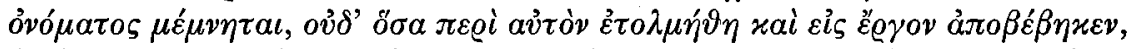

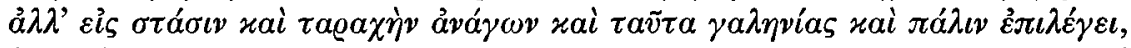

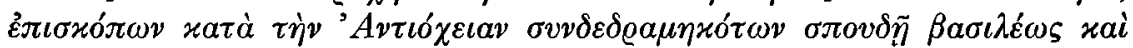

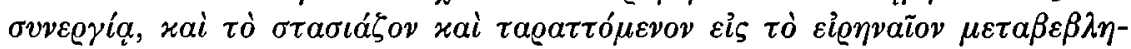

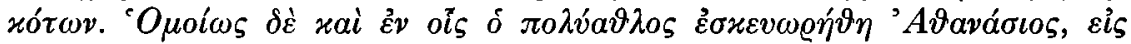

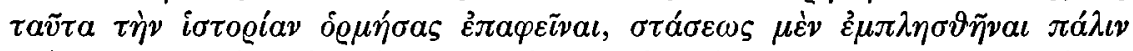

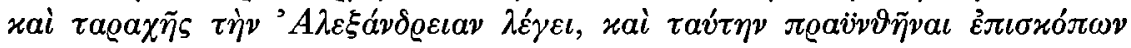

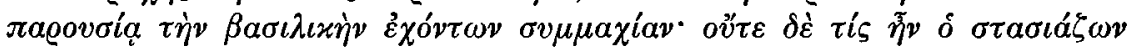

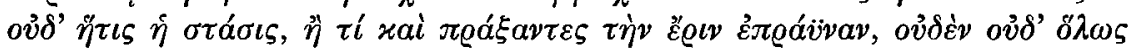

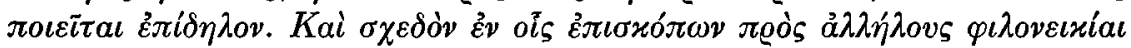

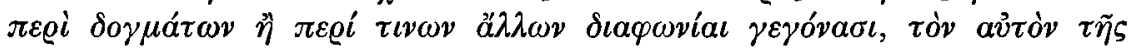

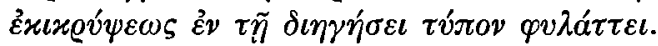

Eine kurze, zu kurze Erwähnung im Carmen Ebedjesu metropolitae Sobae et Armeniae continens catalogum Librorum omnium Ecclesiasticorum, Kap. 11: „Historia Constantini" "1. Immerhin kann daraus geschlossen werden, daß es auch eine syrische Übersetzung der VC gab, von der bislang aber noch keine Handschrift entdeckt ist.

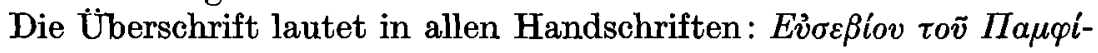

1 J. S. Assemanus, Bibliotheca orientalis III, 1725, 18f. Der Katalog ist keine Übersetzung von De viris ill. des Hieronymus. 


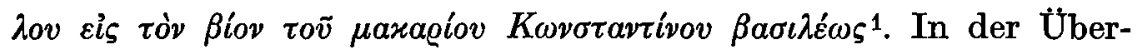
lieferung durch Socrates ist der Titel etwas kürzer gefaßt, und zwar

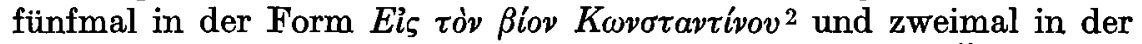

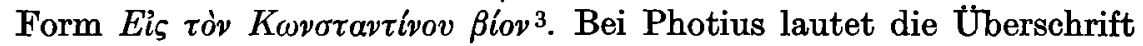

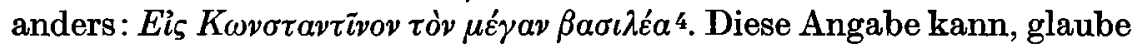
ich, als nicht ursprünglich ausscheiden 5 , denn einmal fehlt das gut bezeugte $\delta$ kios, zum anderen ist die Bezeichnung Konstantins als $\delta$ $\mu \varepsilon \dot{\gamma} \alpha_{\varsigma} \beta a \sigma \iota \lambda \varepsilon \dot{s}$ erst in späterer Zeit gebräuchlich, wie die hagiographischen Viten zeigen ${ }^{6}$. In der Frage, ob die Kurzform des Socrates

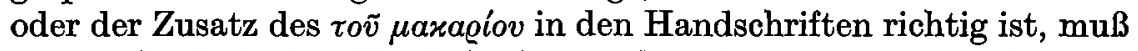
man wohl doch den Handschriften recht geben. Zwar ist zu berücksichtigen, daß Socrates siebenmal denselben Titel - bis auf die oben genannte Wortumstellung - bietet. Anderseits wird Konstantin in den

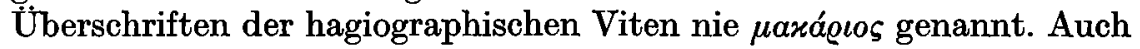
bei anderen Heiligen benutzen die Hagiographen nur selten das Bei-

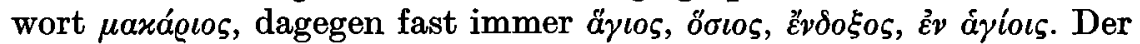
von den Handschriften der VC gebotene Titel kann also kaum in späterer Zeit entstanden sein. Für die Ursprünglichkeit spricht weiter-

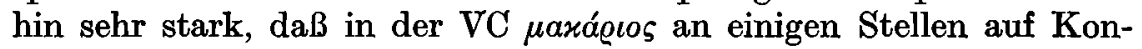
stantin angewendet wird 7 .

\section{Editionen und Obersetzungen}

Der griechische Text der VC wurde zum ersten Mal von Robert Estienne (Stephanus, 1503-1559) in einem durch seine typographische Form berühmten Sammelband der Kirchenhistoriker in Paris 1544 ediert ${ }^{8}$. Die VC folgt hier an zweiter Stelle nach der Kirchengeschichte

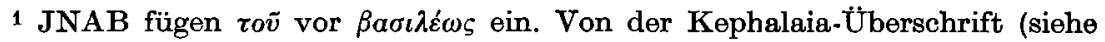
unten S. XLVIff.) können wir hier absehen, da sie eine spätere Zutat ist.

2 Socr. I 1, 2; 8, 20; II 21, 2; V 22, 24; VII 32, 14 .

3 Socr. I 7, 2;8, 4 .

4 Bibl. cod. 127.

5 Heikel, GCS 7, XLV, hielt sie für ursprünglich.

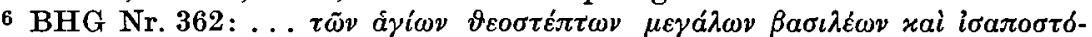

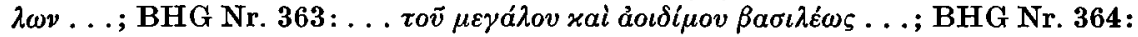

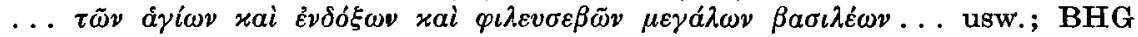

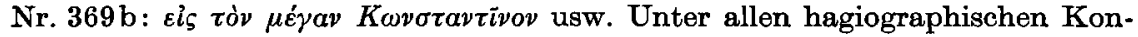
stantinviten habe ich nur vier Ausnahmen gefunden: BHG Nr. 366: $\tau \tilde{\omega} \nu \dot{\varepsilon} \nu$

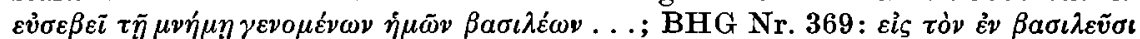

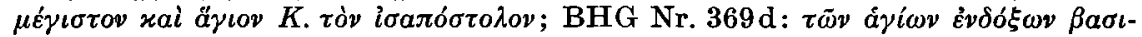

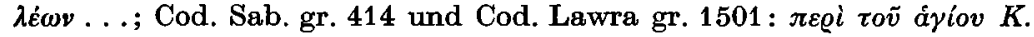

7 Siehe Namenregister.

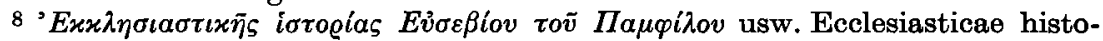
riae Eusebii Pamphili lib. X. Ejusdem de Vita Constantini lib. V usw., Lutetiae Parisiorum 1544, 20, ex officina Roberti Stephani typographi Regii, Regiis typis. Cum privilegio Regis. Fol. 177a-157a enthalten die vier Bücher der 